\title{
EL ESTATUS SOCIAL DE LAS MUJERES Y SU IMPACTO EN LA SALUD DE LA POBLACIÓN
}

\author{
MERCEDES CARRASCO-PORTIÑO \\ Universidad de Alicante \\ Universidad de Concepción (Chile)
}

Recibido: 15/07/2011

Aceptado: 29/09/2011

\section{Referencia del artículo comentado}

KARESTAN C; KOENENN, LINCOLN Alisa y APPLETON Allison. «Women's status and child well-being: a state-level analysis» Social Science \& Medicine, 2006, (63): pp. 2999-3012.

\section{Resumen}

En este estudio se llevó a cabo un análisis ecológico de la relación entre el estado social de las mujeres y el bienestar de los niños en los 50 Estados Unidos. El estado de las mujeres fue determinado mediante 4 índices compuestos: Participación política de las mujeres, autonomía económica, empleo e ingresos, y derechos reproductivos. El bienestar infantil fue medido mediante 5 ítems: porcentaje de niños con bajo peso, mortalidad infantil, mortalidad en adolescentes, tasa de fracaso escolar, y tasa de embarazos infantiles. Los mejores niveles del estado social de las mujeres en todos los indicadores se asociaron significativamente con los mejores niveles de bienestar de los niños en un análisis crudo. Varias asociaciones permanecieron significativas después de ajustar por desigualdades en los ingresos etnia. La participación política de las mujeres se asoció con un porcentaje significativamente menor de niños con bajo peso al nacer $(p<.001)$ y menor tasa de embarazo de adolescentes $(p<.05)$. Los empleos de las mujeres y los ingreso se asociaron con baja mortalidad infantil $(p<.05)$ y de embarazo en adolescentes $(p<.05)$. Mayor autonomía económica y social para las mujeres se asoció con mejores resultados en los niños en todos los indicadores utilizados ( $p<.01$ all). Mayores derechos reproductivos se asociaron con una significativa menor mortalidad infantil $(p<.01)$. Se concluye que el bienestar de los niños es peor en los Estados en 
que las mujeres tienen un menor estado político, económico y social. El estado social de las mujeres es un importante factor del contexto social de los niños, lo cual puede impactar su bienestar. Es necesario desarrollar análisis multinivel de la asociación entre el estado social de las mujeres y el bienestar infantil.

Feminismo/s 18, diciembre 2011, pp. 333-342 


\section{Introducción}

Existe múltiple evidencia de que las desigualdades sociales son un factor estructural determinante de las desigualdades de género en salud 1, 2, 3, 4, 5. Sin embargo, es insuficiente considerar sólo indicadores sociales para captar el estatus de las mujeres respecto a los hombres, como se viene haciendo en estudios sobre desarrollo de género ${ }^{6}$. Es por ello que este ensayo quiere centrarse en un estudio que va más allá de este simplista enfoque. El estudio fue liderado por Karestan Koenen y publicado en la revista Social Science $\mathcal{E}$ Medicine. El objetivo de este estudio fue evaluar la influencia del estatus de las mujeres en el bienestar infantil en cada uno de los 50 estados de los Estados Unidos de Norteamérica. Resulta interesante conocer los elementos que componen el estatus de las mujeres como son: la participación política, el empleo y los ingresos, la autonomía económica y social, y los derechos reproductivos.

Cabe destacar que los valores de salud globales de los países no proporcionan información precisa sobre las diferencias o desigualdades en la distribución geográfica de la salud y enfermedad, y sus determinantes socioeconómicos, políticos y culturales; siendo necesario considerar unidades geopolíticas más pequeñas ${ }^{7,8,9,10}$, como se ha considerado en el estudio de Koenen et al.

1. KaWAChi, I; Kennedy, B. P.; GuPta, V.; Prothrow-Stith, D. Women's status and the health of women and men: a view from the States. Soc Sci \& Med (1999), pp. 21-32.

2. MetCAlfe, B. D.; Afanassieva, M. Gender, work, and equal opportunities in central and eastern Europe. Women in Management Review (2005), pp. 397-411.

3. YU, Mei-Yu; SARRY, Rosemary. Women's Health Status and Gender Inequality in China. Soc Sci Med (1997), pp. 1885-1898.

4. Muntaner, C., Borrell, C.; Vanroelen, C.; Chung, H.; Benach, J.; Kim, I. H.; NG, E. Employment relations, social class and health: a review and analysis of conceptual and measurement alternatives. Soc Sci Med. (2010), pp. 2130-2140.

5. Artacoz, L; Cortes, I; Borrell, C; Escribà-Agüir, V; Cascant, L. Gender and social class differences in the association between early retirement and health in Spain. Women's Health Issues. (2010), pp. 441-447.

6. YU, Mei-Yu; SARRY, Rosemary. Op. cit., p. 1.

7. KaWAChi, I.; Kennedy, B. P.; Gupta, V.; Prothrow-Stith, D. Op. cit. p. 2.

8. StARFIELD, B. State of the art in research on equity in health. J Health Polit.Policy Law. (2006), pp. 11-32.

9. WilKInSON, R. G.; PICKETT, K. E. Income inequality and population health: A review and explanation of the evidence. Soc Sci Med. (2006), pp. 1768-1784.

10. StarfiELD, B. Pathways of influence on equity in health. Soc Sci Med. (2007), pp. 1355-1362.

Feminismo/s 18, diciembre 2011, pp. 333-342 
Desde la Conferencia de Beijing en 1995 y el informe Beijing +5, la Organización de Naciones Unidas viene destacando la necesidad de adoptar indicadores de género para diagnosticar la situación de las mujeres y hacer políticas públicas consecuentes ${ }^{11,12}$. Ha sido tal la importancia de la sensibilidad de género en el desarrollo que se ha incorporado en el $3^{\text {er }}$ Objetivo de Desarrollo del Milenio de Naciones Unidas, que pretende eliminar las desigualdades de género e incrementar la autonomía de las mujeres en el año $2015^{13,14,15}$. Es por ello, que se hace necesario la reflexión acerca de la construcción de indicadores que midan la situación de las mujeres en una sociedad y su impacto en la salud de la población como se ha hecho en el estudio de Koenen et al.

\section{Desarrollo}

Koenen y su equipo quisieron seguir la metodología de trabajo de Kawachi et al. ${ }^{16}$ y McLeod et al. ${ }^{17}$, llegando a elaborar un estudio de diseño ecológicotransversal que intentaba explorar la relación entre el estatus social de las mujeres y el bienestar de niños/niñas en Estados Unidos.

11. NACIONES UNIDAS. Informe de la Cuarta Conferencia Mundial sobre la Mujer. Disponible en: www.un.org/womenwatch/confer/beijing/reports/platesp.htm (Acceso 24.07.2011).

12. NACIONES UNIDOS BEIJING+5. Período extraordinario de la Asamblea General. Mujeres 2000: Igualdad de género, desarrollo y paz para el siglo XXI. Nueva York: 2000. Disponible en: www.un.org/spanish/conferences/Beijing/mujer2021.htm (Acceso 24.07 .2011$)$

13. NACIONES UNIDAS. Objetivos de Desarrollo del Milenio. Disponible en: http://www. undp.org/spanish/mdg/basics.shtml (Acceso 24.07.20114).

14. GLOBAL MONITORING REPORT 2007. Chapter 3. Promoting Gender Equality and Women's Empowerment. Disponible en: http://siteresources.worldbank.org/INTGLOMONREP2007/Resources/3413191-1176390231604/105-148_GMRch3.pdf (Acceso 24.07.2011)

15. THE WORLD BANK. Global Monitoring Report 2007. Confronting the Challenges of Gender Equality and Fragile States. Fact Sheet: Promoting Gender Equality and Women's Empowerment. Disponible en: http://web.worldbank.org/WBSITE/EXTERNAL/EXTDEC/EXTGLOBALMONITOR/EXTGLOMONREP2007/0,, menuPK: 3413296 pagePK:64218926 piPK:64218953 theSitePK:3413261,00.html (Acceso 24.07.2011).

16. KaWachi, I.; Kennedy, B. P.; Gupta, V.; Prothrow-Stith, D. Op. cit. p. 2.

17. MCleod, J. D.; NONNEMAKeR, J. M.; CAll, K.T. Income inequality, race, and child well-being: An aggregate analysis in the 50 United States. Journal of Health \& Social Behavior (2004), pp. 249-264. 
¿De donde recogieron la información para valorar el bienestar infantil?

El bienestar de los niños y las niñas se evaluó a través de los indicadores de bienestar y salud infantil de la Fundación Annie E. Casey ${ }^{18}$, que son los siguientes:

- Bajo peso al nacer.

- Tasa de mortalidad infantil.

- Tasa de mortalidad de adolescentes por homicidio, suicidio y accidentes (15 a 19 años).

- Tasa de natalidad en adolescentes (15 a 17 años).

- Abandono escolar de secundaria (16 a 19 años).

¿Cuál fue la fuente de información de los indicadores del estatus de las mujeres?

Los indicadores sobre el status de las mujeres en la sociedad se extrajeron del Institute for Women's Policy Research (IWPR) ${ }^{19}$ que calculan anualmente indicadores que miden la participación política, el empleo y los ingresos, la autonomía social y económica, y por último los derechos reproductivos de las mujeres (Ver tabla 1).

En este estudio se realizaron análisis de los datos controlando por variables como el ingreso medio familiar, el porcentaje de la población en estado de pobreza, la desigualdad en ingresos y composición racial. Los resultados demuestran la relación que existen entre el estatus de las mujeres y los indicadores de bienestar infantil estudiados. En la siguiente tabla 2 se muestra un resumen de las asociaciones en el estudio motivo de este ensayo.

¿Cuál será el impacto del estatus de las mujeres en la salud de la población española? ¿Se podría realizar un estudio de similares características en España?

Actualmente, no disponemos de un centro tan especializado como el Institute for Women's Policy Research de Estados Unidos. Lo más cercano es el Instituto de la Mujer del Ministerio de Sanidad, Política Social e Igualdad. Sin embargo, dentro del grupo de investigación de la Universidad de Alicante se está

18. ANNIE E. CASEY FOUNDATION. Kids count data book: 2004. Baltimore, MD: Annie E. Casey Foundation, 2004.

19. INSTITUTE FOR WOMEN'S POLICY RESEARCH. The Status of Women in the States. Disponible en http://www.iwpr.org/publications/pubs/the-status-of-women-in-thestates (Acceso 24.07.2011)

Feminismo/s 18, diciembre 2011, pp. 333-342 
Tabla 1. Indicadores del estatus de las mujeres y sus componentes según el Institute for Women's Policy Research

\begin{tabular}{|c|c|}
\hline Indicador & Componente \\
\hline \multirow{4}{*}{$\begin{array}{l}\text { Índice de } \\
\text { participación } \\
\text { política }\end{array}$} & Mujeres con derecho a voto \\
\hline & Mujeres que han ejercido el voto \\
\hline & $\begin{array}{l}\text { Mujeres en puestos políticos-legislativos estatales (senadoras, } \\
\text { diputadas, entre otros) }\end{array}$ \\
\hline & Recursos institucionales para mujeres \\
\hline \multirow{4}{*}{$\begin{array}{l}\text { Índice de empleo e } \\
\text { ingresos }\end{array}$} & Ingreso anual medio de las mujeres \\
\hline & Razón de ingresos de mujeres respecto a los hombres \\
\hline & Mujeres con participación en la fuerza de trabajo \\
\hline & Mujeres con cargos directivos y ocupaciones profesionales. \\
\hline \multirow{4}{*}{$\begin{array}{l}\text { Índice de } \\
\text { autonomía social y } \\
\text { económica }\end{array}$} & Porcentaje de mujeres con seguridad social \\
\hline & $\begin{array}{l}\text { Porcentaje de mujeres de } 25 \text { o más años con } 4 \text { o más años de } \\
\text { universidad. }\end{array}$ \\
\hline & Mujeres con trabajo propio privado \\
\hline & Porcentaje de mujeres por debajo del nivel de pobrezas \\
\hline \multirow[t]{8}{*}{$\begin{array}{l}\text { Índice de derechos } \\
\text { reproductivos }\end{array}$} & $\begin{array}{l}\text { Consentimiento obligatorio para la interrupción voluntaria del } \\
\text { embarazo (IVE) en menores de edad }\end{array}$ \\
\hline & Períodos de espera para la IVE \\
\hline & Restricciones de instituciones pública para realizar la IVE \\
\hline & $\begin{array}{l}\text { Porcentaje de mujeres que viven en condados con una legislación } \\
\text { que facilita la IVE }\end{array}$ \\
\hline & Gobernadores(as)/Legisladores(as) a favor de la IVE \\
\hline & Tratamientos de fertilidad \\
\hline & Matrimonios homosexuales y adopción \\
\hline & Educación sexual obligatoria \\
\hline
\end{tabular}

Tabla 2. Asociación de los indicadores de situación/estatus de la mujer con el bienestar infantil estadísticamente significativa (modelos ajustados)

\begin{tabular}{|l|c|c|c|c|c|}
\hline & $\begin{array}{c}\text { Bajo peso al } \\
\text { nacer }\end{array}$ & $\begin{array}{c}\text { Mortalidad } \\
\text { infantil }\end{array}$ & $\begin{array}{c}\text { Mortalidad } \\
\text { adolescente }\end{array}$ & $\begin{array}{c}\text { Abandono } \\
\text { escolar } \\
\text { secundaria }\end{array}$ & $\begin{array}{c}\text { Tasa de } \\
\text { natalidad en } \\
\text { adolescente }\end{array}$ \\
\hline $\begin{array}{l}\text { Participación } \\
\text { política }\end{array}$ & $\mathrm{X}$ & $\mathrm{X}$ & & $\mathrm{X}$ \\
\hline $\begin{array}{l}\text { Empleo e } \\
\text { ingresos }\end{array}$ & $\mathrm{X}$ & $\mathrm{X}$ & $\mathrm{X}$ & $\mathrm{X}$ & $\mathrm{X}$ \\
\hline $\begin{array}{l}\text { Autonomía } \\
\text { económica y } \\
\text { social }\end{array}$ & & $\mathrm{X}$ & & & \\
\hline $\begin{array}{l}\text { Derechos } \\
\text { reproductivos }\end{array}$ & & & & & \\
\hline
\end{tabular}


llevando a cabo un proyecto que pretende construir indicadores compuestos de desarrollo de género y de la situación social de hombres y mujeres a nivel de comunidades autónomas para facilitar la exploración de asociaciones entre estos indicadores con problemas de salud y sus determinantes.

En España, existe un trabajo que mediante el índice de desarrollo de género propuesto por la Organización de Naciones Unidos en el año 1995, se evidenció que las provincias españolas con menor desarrollo de género presentaban mayores tasas de mortalidad por violencia del compañero íntimo ${ }^{20}$. Este indicador ha sido reemplazado en el 2010 por el índice de desigualdad de género. Lo que hace indispensable pensar en la construcción de este y otros indicadores para la valoración de la situación social de mujeres como también de hombres. Por otro lado, es importante destacar que en un anterior estudio liderado por Ichiro Kawachi en Estados Unidos se demostró con los datos del Institute for Women's Policy Research que el estatus de las mujeres puede predecir el estado de salud de los hombres también ${ }^{21}$, no sólo de niños(as) y de su propia salud.

Cabe destacar que el Institute for Women's Policy Research (IWPR), con sede en Washington fue fundado el 1987 por la necesidad de disponer de una organización cuyo propósito fuera evidenciar la injusticia económica que vivían las mujeres estadounidenses. Sin embargo, después de más de 25 años, este instituto ha permitido que políticos y líderes claves de la sociedad estadounidense hablen un idioma común a través de la información producida por éste. En este sentido, ha permitido aumentar la capacidad de formular y aplicar leyes que beneficien a las mujeres y sus familias en este país (Ver figura 1).

El IWPR ha publicado cientos de informes, notas informativas y de investigación donde la mujer es el punto central del análisis, y aunque sólo produzcan información de Estados Unidos son un ejemplo a imitar para el resto del mundo, contribuyendo así, a mejorar la investigación y las políticas basadas en la evidencia para disminuir las desigualdades de género.

\section{Conclusiones}

Un grave problema que compromete a las investigaciones poblacionales es la falta de información para estimar la relación entre determinantes de contexto

20. Vives-Cases, C.; Álvarez-Dardet, C.; Carrasco-Portiño, M.; Torrubiano-DomínGUEZ, J. El impacto de la desigualdad de género en la violencia del compañero íntimo en España. Gac Sanit. (2007), pp. 242-246

21. KaWachi, I.; Kennedy, B. P.; Gupta, V; Prothrow-Stith, D. Op. cit. p. 2

Feminismo/s 18, diciembre 2011, pp. 333-342 


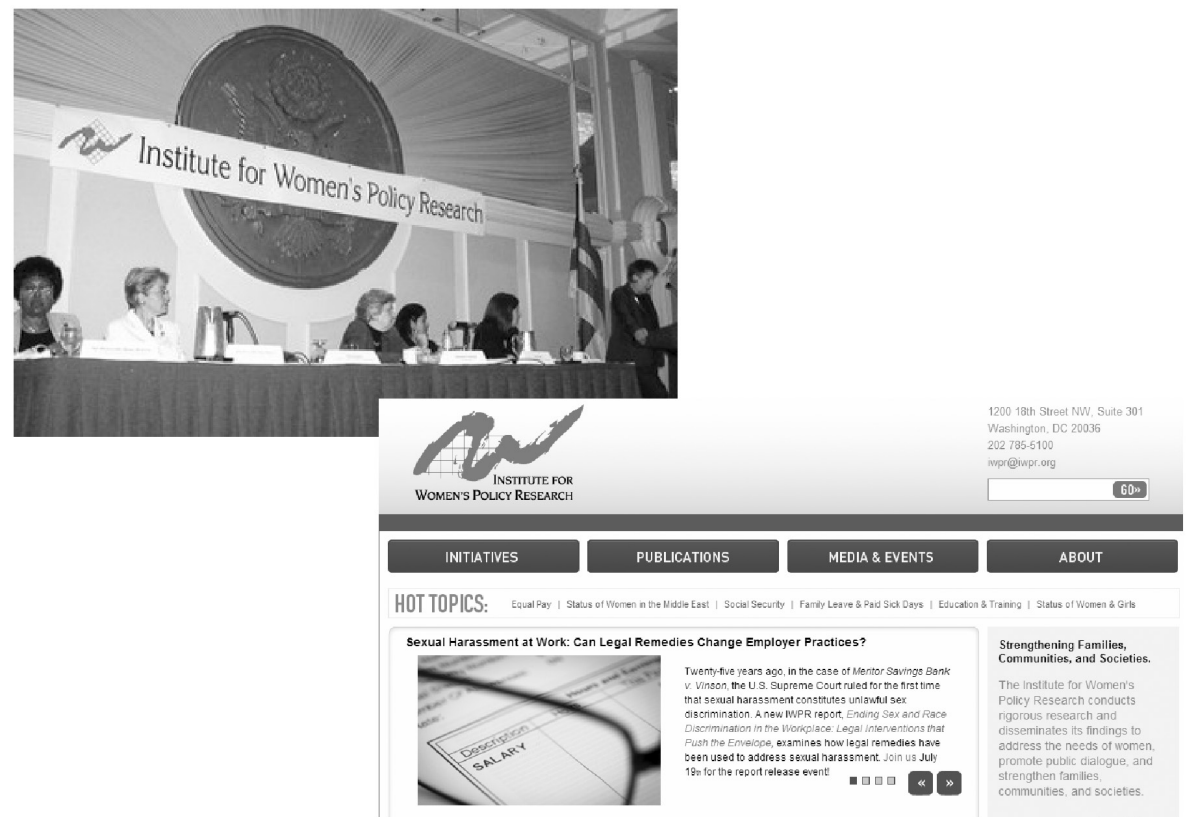

Figura 1.

social y los problemas de salud y sus estrategias terapéuticas. Por otro lado, la igualdad de género no siempre es un elemento de los programas de desarrollo económico y social, en parte, por la falta de evidencia científica sobre su relevancia para el bienestar, la calidad de vida y la salud.

Afortunadamente en España, la Ley Orgánica para la igualdad efectiva de mujeres y hombres en 2007 favorece la producción, recolección y sistematización de datos con el fin de hacer viables y aplicables políticas de desarrollo de género. Esto sucede en el marco de iniciativas internacionales para la igualdad entre las mujeres y los hombres, en donde los indicadores nacionales y transnacionales pasan a tener reconocimiento o proyección social y política, al acordar los diferentes países objetivos comunes a partir de indicadores de control.

El desarrollo de políticas y estrategias efectivas de mejora de la situación socio-sanitaria en las comunidades autónomas y provincias de España precisa de un esfuerzo de compilación de información que facilite la creación de indicadores compuestos de desarrollo y por ende de desigualdades de género, para analizar su efecto en la salud de ambos sexos. 


\section{Referencias bibliográficas}

ANNIE E. CASEY FOUNDATION. Kids count data book: 2004. Baltimore, MD: Annie E. Casey Foundation, 2004.

Artacoz, L.; Cortes, I.; Borrell, C.; EscribÀ-AgüIr, V.; CASCANT, L. Gender and social class differences in the association between early retirement and health in Spain. Women's Health Issues. (2010), pp. 441-447.

DomínguEZ, J. El impacto de la desigualdad de género en la violencia del compañero íntimo en España. Gac Sanit. (2007), pp. 242-246

GLOBAL MONITORING REPORT 2007. Chapter 3. Promoting Gender Equality and Women's Empowerment. Disponible en: http://siteresources.worldbank. org/INTGLOMONREP2007/Resources/3413191-1176390231604/105-148_ GMRch3.pdf (Acceso 24.07.2011)

INSTITUTE FOR WOMEN'S POLICY RESEARCH. The Status of Women in the States. Disponible en http://www.iwpr.org/publications/pubs/the-status-ofwomen-in-the-states (Acceso 24.07.2011)

KaWAchi, I.; Kennedy, B. P.; Gupta, V.; Prothrow-Stith, D. Women's status and the health of women and men: a view from the States. Soc Sci \& Med (1999), pp. 21-32.

MCleOd, J. D.; NONNEMAKer, J. M.; CALL, K.T. Income inequality, race, and child well-being: An aggregate analysis in the 50 United States. Journal of Health \& Social Behavior (2004), pp. 249-264.

MetCalfe, B. D.; Afanassieva, M. Gender, work, and equal opportunities in central and eastern Europe. Women in Management Review (2005), pp. 397-411.

Muntaner, C., Borrell, C.; Vanroelen, C.; Chung, H.; BenaCH, J.; Kim, I. H..; NG, E. Employment relations, social class and health: a review and analysis of conceptual and measurement alternatives. Soc Sci Med. (2010), pp. 2130-2140.

NACIONES UNIDAS. Informe de la Cuarta Conferencia Mundial sobre la Mujer. Disponible en: www.un.org/womenwatch/confer/beijing/reports/platesp.htm (Acceso 24.07.2011).

NACIONES UNIDOS BEIJING+5. Período extraordinario de la Asamblea General. Mujeres 2000: Igualdad de género, desarrollo y paz para el siglo XXI. Nueva York: 2000. Disponible en: www.un.org/spanish/conferences/Beijing/ mujer2021.htm (Acceso 24.07.2011).

NACIONES UNIDAS. Objetivos de Desarrollo del Milenio. Disponible en: http:// www.undp.org/spanish/mdg/basics.shtml (Acceso 24.07.20114).

StARFIEld, B. Pathways of influence on equity in health. Soc Sci Med. (2007), pp. 1355-1362.

STARFIELD, B. State of the art in research on equity in health. J Health Polit.Policy Law. (2006), pp. 11-32. 
THE WORLD BANK. Global Monitoring Report 2007. Confronting the Challenges of Gender Equality and Fragile States. Fact Sheet: Promoting Gender Equality and Women's Empowerment. Disponible en: http://web.worldbank. org/WBSITE/EXTERNAL/EXTDEC/EXTGLOBALMONITOR/EXTGLOMON REP2007/0,,menuPK:3413296 pagePK:64218926 piPK:64218953 theSite PK:3413261,00.html (Acceso 24.07.2011).

Vives-Cases, C.; Álvarez-Dardet, C.; Carrasco-Portiño, M.; Torrubiano.

Wilkinson, R. G.; PiCKeTt, K. E. Income inequality and population health: A review and explanation of the evidence. Soc Sci Med. (2006), pp. 1768-1784. YU, Mei-Yu; SARRY, Rosemary. Women's Health Status and Gender Inequality in China. Soc Sci Med (1997), pp. 1885-1898. 